\title{
Identification of Offenders by Mind Fingerprinting Technology
}

\author{
Ch Sudhakar ${ }^{1}$ and N.Thirupathi Rao $^{2}$ \\ Department of Computer Science \& Engineering, Vignan's Institute of Information \\ Technology (A), Visakhapatnam, AP, India \\ ${ }^{1}$ sudhakarcheetirala@gmail.com
}

\begin{abstract}
Mind Fingerprinting has recognized a hundred percentages precise in over 120 investigations with trials on FBI mediators, tests aimed at a United States intelligence agency and United States Navy, and trials on real-life situations containing offences. The Mind Finger Printing (MFP) detect hidden facts stored in the minds by measuring mind wave responses. We were compared P300-MERMER ("Memory and Encoding Related Multifaceted Electroencephalographic Response") and P300 result associated mind capacities for fault rate laccurateness and numerical assurance in four real studies. 76 tests discovered existence or nonappearance of info concerning (1) real-life proceedings as well as offence crime; (2) actual crime with significant penalty (3) facts distinctive to Federal Bureau of Investigation (FBI) agents and (4) facts inimitable to explosive Bomb disposal experts. Among together P300-MERMER and P300, the faulty rate was zero percentage: calculations were a hundred per cent perfect, no false positive or false negative; and no in calculations. Counter procedures have no outcome. Average statistical assurance for calculations was 99.9 \% among P300-MERMER and $99.6 \%$ among P300. Mind fingerprinting method and technical values for the research laboratory and turf applications were deliberated. Crucial dissimilarities in techniques that generate different results are recognized. Noticeably diverse techniques in further studies have formed over 10 time's refined faulty rates and noticeably inferior statistical confidence. Facts maintain the assumptions to facilitate accurateness, consistency, and legality on subsequent the mind fingerprinting technical values outline in this.
\end{abstract}

Keywords: Mind, Finger printing, P300, MERMER

\section{Introduction}

This research analyzed the precision, and unwavering quality of the Memory and Encoding Interrelated Multifaceted Electroencephalographic Response (MERMER) method for identifying data associated to occasion's subjects have encountered, despite subjects' endeavour's to hide that learning. Data acquired over meetings were utilized to create incitement sets comprising of words and phrases displayed to subjects outwardly by PC. Sets were made out of three types of incentives: educational experience-related (Probes), stimuli the subject was requested to memorize and respond to (Targets), and insignificant data (Irrelevant). Each procedure of incentive was tried on two people: (1) one person who had taken an interest in the occasion being referred to and accordingly required the appropriate

Article history:

Received (April 15, 2020), Review Result (May 17, 2020), Accepted (June 21, 2020) 
data stored in their mind and (2) one who required not. Totally six subjects were verified. Electrical mind reactions to the stimuli were noted non- obtrusively from the scalp and investigated. Members, (memory and encoding related multifaceted electroencephalographic responses), of which the P300 is a sub-segment, were used to decide if the subject had the pertinent data put away in his or not [1][2][3].

In the area of crime department, a novel lie discoverer has-been matured in the USA called "Mind fingerprinting" [4]. This detection is invented to be the finest lie discoverer accessible as going on a date and is supposed to notice still soft criminals. They exceed the lie discoverer test with no trouble. The new technique employs mind waves, which help detect whether the one exposed to the test, recollects better details of the crime. Still, if the individual gladly suppresses the needed info, the mind wave is sure to ensnare him, affording to the specialists who are keyed up regarding the block. Mind Fingerprinting is considered to decide whether an entity recognizes precise info associated with an occurrence or action by measure electronic mind wave response to pictures, words or phrases accessible on a CPU. The method can be used merely in a situation wherever agents contain the inadequate quantity of exact info regarding an activity that would be identified just to investigators [5]. In this esteem, Mind Fingerprinting is measured a kind of responsible fact Test, where the responsible gathering is likely to respond robustly to the related aspect of the experience of action.

The polygraph actions to assess the strength of suspect responsible facts rely on the extent of autonomic palm sweating and heart rate, etc., whereas Mind Fingerprinting dealings electronic mind actions via built-in headband sensors. Mind Fingerprinting is supposed to be further precise in detecting responsible fact separates of the false positives of conventional detector techniques; however, this is ardently undecided by expert scientists.

\section{Narration of " $m$ ind fingerprinting"}

It is founded on the significant article of corresponding approximately on the individual of the suspicious through approximately from the crime section. It contests prints at the crime scene with patterns on the fingers of the suspects [6]. DNA fingerprinting contests organic samples from the crime scene with biological samples from the suspects, and it contests data deposited in the mind of the suspicious with info from the crime section. Mind fingerprinting training analyses the facts constructed on the P300-MERMER.

In the early mind fingerprinting inspect, Mr Farwell and Mr Don Chin applied the P300 result associated mind prospective. Later both revealed that theP300 could be measured to be a division of a superior reaction called "Memory and Encoding Related Multifaceted Electroencephalographic Response" (MERMER). In the 1990s, Mr Farwell and Mr Drew Richardson have performed the mind fingerprinting study on Federal Bureau of Investigation representatives, $\mathrm{P} 300$ suspension of 600 to $700 \mathrm{~ms}$ remained generally originate in research.

Wherever the stimulus was info annoying and the intellectual giving out necessary was considerable. At that instance, in such investigation, a first incentive was usually accessible each 1000 to $1500 \mathrm{~ms}$. In handling with actual life situations, Mr Farwell and Richardson create it needed to use more and more extended compound incentives to precisely connect the essential info to the issue. In classify to here practical stimulus that correctly characterizes unique knowledge to FBI representative, they originate it needed to use stimulus containing several disputes each. To provide the focus instance to practice the stimulus and take action appropriately, the time between stimulus from 1500 to $3000 \mathrm{~ms}$. Reconfirmation a more extended section of mind wave data in every trial [7][8]. 


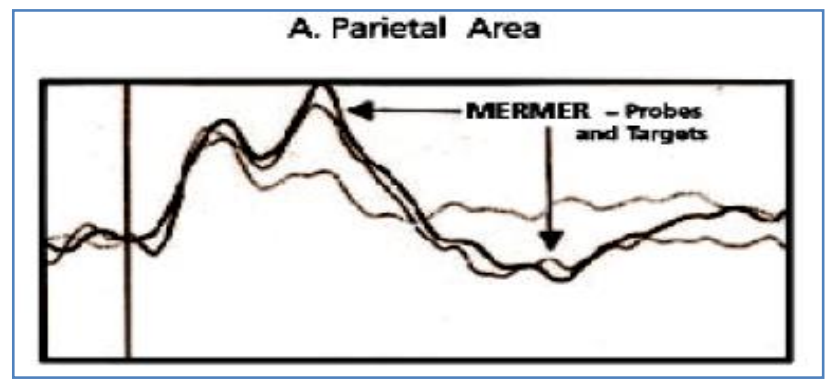

Figure 1(a). Mind wave

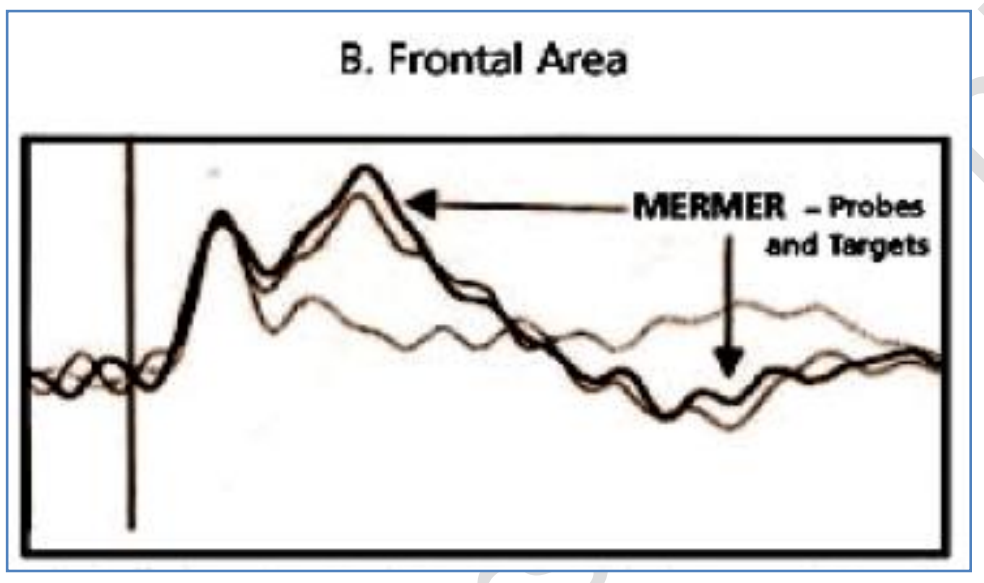

Figure 2(b). Mind wave

\section{Obligation of mind fingerprinting}

It is based on the standard that the mind is essential to all social actions. In a criminal action, around might or might not be various categories of marginal indications, but the mind is continuously around, scheduling, implementing, and footage the crime. The essential variances of amongst criminal and a misleadingly suspect. It identifies systematically. The secrets of Mind Fingerprinting Matching proof at the crime scene with the mind [9][10]. Once a crime is dedicated, a record is stored in the mind of the offender. Mind Fingerprinting delivers resources to accurately and technically associate proof from the criminal actions with proof stored in mind.

[Figure 2] describing the technique used is related to the "Guilty Information Test", a sequence of words, sounds, or pictures are accessible via computer to the subject for a segment of a second respectively. Respectively of these incentives are structured. The goal incentives remain preferred to be appropriate data to the verified subject. They are used to create a standard mind reaction for data that is important to the subject existence verified. Furthermost the non-target incentives are inappropriate and are isolated to the situation. The unrelated incitements do not cause a MERMER and establish responsibility for data that is irrelevant to the subject. Approximately the non-target applies to the condition that the subject is presence verified for. These are related to the test then are essential to the subject. A subject requiring data in their mind and the answer to the Investigation stimulus will be vague from the unrelated stimulus. 


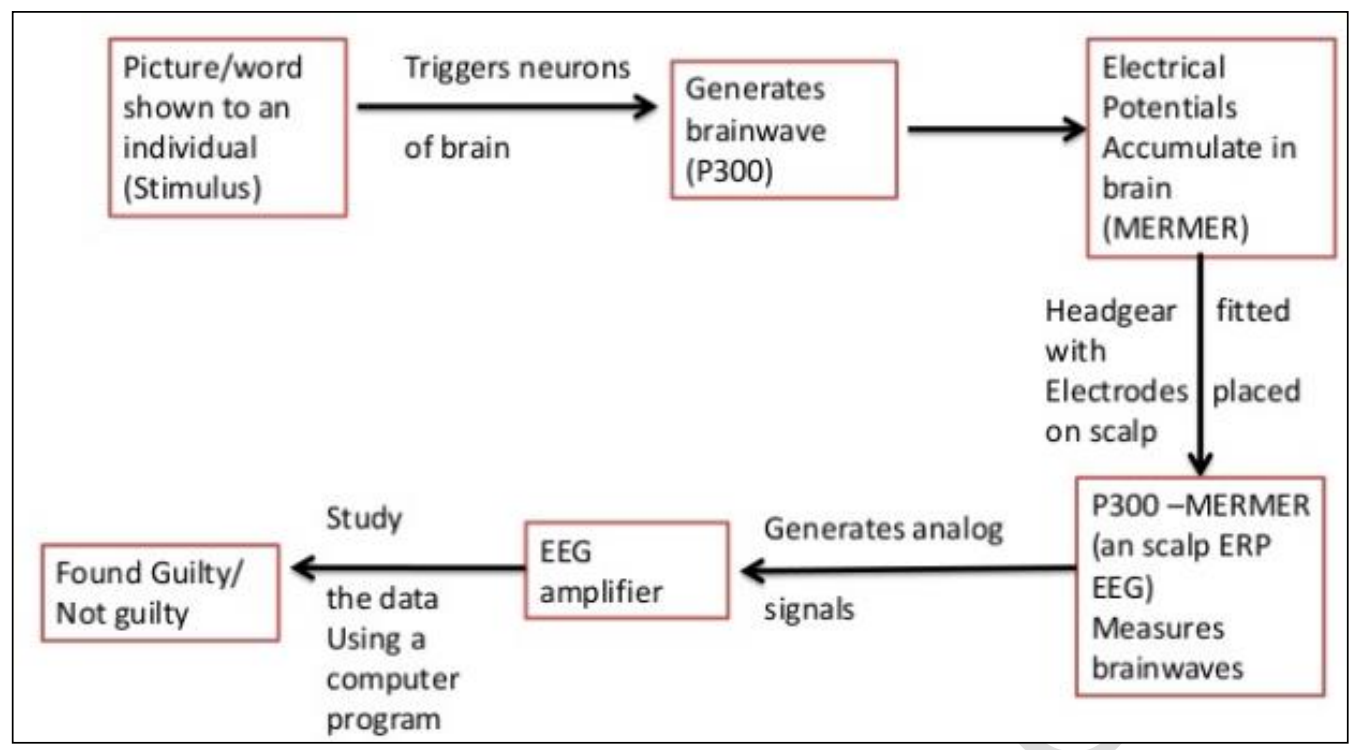

Figure 2. Method of finding guilty

Every knowledge includes skill and judgment on the number of its specialists, and the knowledge of Mind Fingerprinting challenging is no segregation. Agents 'necessity for careful, scientific resources of including agents through crime scene proof has motivated some scientists to invite. The forensic department is continuously developing, from the finding of the individuality of the human fingerprint to the ability to corresponding a criminal to their crime through DNA profiling. But fingerprint and DNA authorization are unadorned in the individual one per cent of all particular belongings.

All criminal leaves are proof in the final. The contribution is to recognize in what way to find it. The advanced procedure is testing a way of selecting the respondent's astuteness to twist the offender's specific retention counter to him.

\section{System architecture:}

[Figure 3] summaries the steps of data acquirement and investigation in mind fingerprinting. There are three types of incentives offered: targets, investigations and irrelevant. Investigations hold info that applies to the crime or other inspected situation. Investigations have three essential characteristics.

- Investigations hold features of the crime that in the verdict of the criminal agent, the offenders would require experts in obligating the crime.

- Investigations have info that the topic has not at all of expressive if he did not contribute to the crime.

- Investigations hold info that the topic privileges not to identify or to distinguish as crucial for any purpose.

- Software established by the Mind Fingerprinting research laboratory for the facts attainment and investigation. 


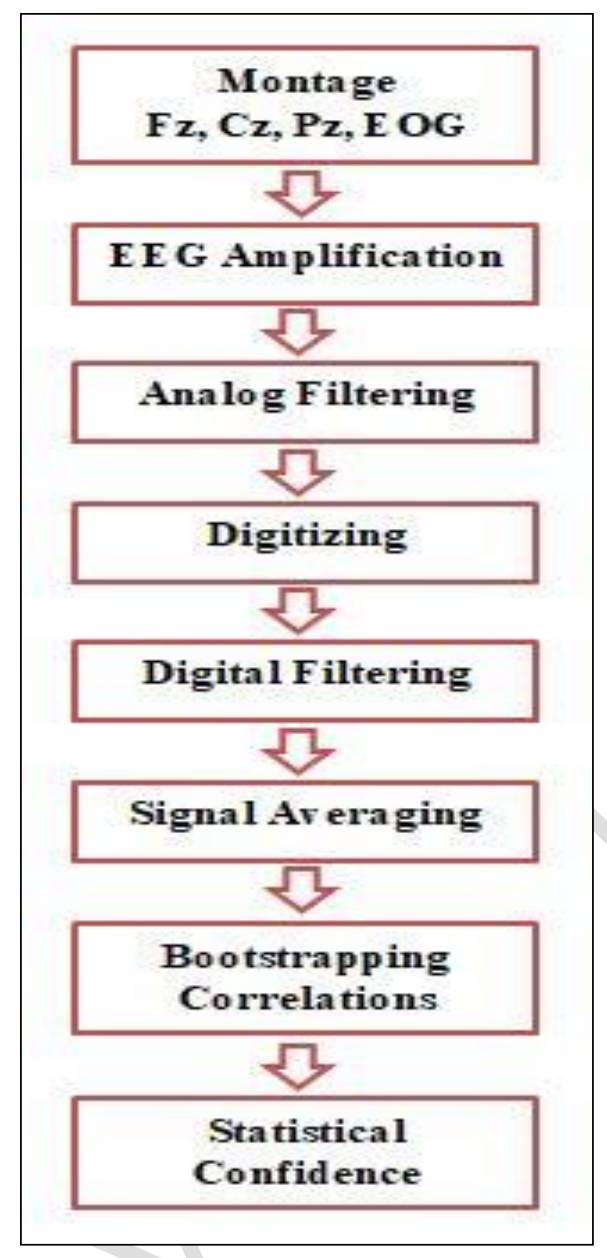

Figure 3: System architecture

The technical question raised by a mind fingerprinting test is whether the topic is expert concerning the corruption, crime or inspected condition. Explicitly, the serious mutable is his acknowledgement of the info contained in the investigations as substantial in the situation of the crime. It is expected that the investigations will cause a P300-MERMER. The morphology, heft and potential will be representative of the different question's reply to such incentives when the subject recognizes the appropriate info. For a question who is expert or info existent," the investigations hold info relating identified features of the crime and for who is "info absent," the investigations hold info relating reasonable structures of the crime that are not predictable to be correct. To accurately classify the investigation answers into one of these two classifications, it is required to separate the serious variable. To achieve this, two values are essential: an ordinary for the answer of this topic to incentives covering recognized features of the crime, and atypical for the answer of this topic to incentives covering reasonable but unidentified features of the offence, crime, corruption, etc.

\section{Stages of mind fingerprinting}

In "DNA fingerprinting and fingerprinting" the indication predictable and composed at the crime scene, and conserved correctly until a doubtful is captured and associated with 
indication on the individual of the suspicious to identify a contest to suspicious at the crime scene.

There are four phases of Mind Fingerprinting, which are related to the DNA fingerprinting:

1. Crime Scene Evidence Collection: In this phase, an proficient in Mind Fingerprinting inspects the crime scene and further proof associated with the crime to recognize particulars of the crime.

2. Mind Evidence Collection: The proficient then manners the Mind Proof Gathering in demand to control whether or not the proof after the crime section competitions proof stowed in the mind of the suspicious.

3. Computer Mark Investigation: In this, the classification makes a scientific resolve as to whether or not this detailed proof is stowed in mind, and calculates a numerical assurance for that purpose.

4. Scientific Result: This purpose and statistical assurance organize the Systematic Outcome of Mind Fingerprinting are the particulars of the corruption, offence, crime are stored in the mind of the suspicious, or the particulars of these are not stored in the mind of the suspicious.

Scientific principles for Mind Fingerprinting Tests:

The following processes encompass the technical principles for mind fingerprinting tests are as follows:

1. Practice methods and equipment for incentive performance, facts and data recording as well as an acquisition that are contained by the principles for the arena of perceptive psychophysiology and incident associated mind possible investigation. These principles are fine recognized somewhere else. For instance, the typical measures, Mr Farwell hosted as an indication in the Harrington case was recognized by the law court, the technical magazines, and the further professional observers in the case. Use a footage period long sufficient to contain the complete P300-MERMER. For pictographic incentives or excellent word incentives, uses at least a 1,800-ms footage time.

2. Usage accurate probe situation. The P300-MERMER and P300are generally identified to be Pz in the standard Test 10-20 System, most at the midline parietal scalp position.

3. Relate mind fingerprinting checks individual after there is enough info that is recognized one to the culprit and agents. Use a min of six investigations and targets.

4. Usage incentives that segregate the serious adjustable: the question information or lack of facts of the analysis incentives as substantial in the situation of the inspected state. Acquire the pertinent facts from the immoral detectives. Split the related information into investigates incentives and stimuli objectives. Investigate incentives represent info that has not stayed exposed to the subject. Extrinsic incentives contain pieces of evidence that have been exposed to the focus after the corruption, offence, crime or investigated condition.

5. If initially, there are smaller quantity marks than probes, produce more objectives. Preferably, this is completed by looking for other known evidence from the criminal detectives. Otherwise, some prospectives investigate incentives can be used as objectives by revealing to the issues of the individual items.

6. For each investigation and respectively objective, formulate numerous incentives of the same type that are disparate to the inspected situation. These become unrelated incentives. Use incentives that separate the critical adaptable. For unrelated incentives, select objects that would be correspondingly reasonable for non-knowledgeable questions. The incentive ratio is about one-sixth investigations, one-sixth objectives, and two-thirds inappropriate.

7. Determine that the analyses hold evidence that the questions have no identified method of significant, other than contribution in the inspected condition. These facts are providing by 
the immoral detective for field training and outcomes from relevant facts mechanism in laboratory training.

8. Make confident that the topic recognizes the importance of the investigations, and make sure that the analyses represent only facts that the question denies significant. Illustrate the importance of respectively investigate the question-indication him the investigation and the equivalent unrelated, deprived of enlightening, which is the investigation.

9. If an issue has an awareness of any investigations for a purpose unconnected to the inspected condition, remove these from the incentive set. This delivers the focus with a chance to reveal any awareness of the investigations that he may require for any solid object formerly unfamiliar to the expert. These determinations stop any non-incriminating facts from presence incorporated in the test.

10. Ascertain that the subject knows the targets and their significance in the context of the investigated situation. Show him a list of the targets. Describe the significance of each target to the subject.

11. Need a specific behavioural task that needs the subject to be familiar with and method each motivation, particularly together with the investigate incentives, and to evidence behaviorally that he has completed so on each test.

12. Educate the questions to compress unique key in rejoinder to marks, and an additional switch in reaction to all further incentives. Do not educate the focuses to "recline" or "express the veracity" in retort to provocations.

13. The necessary amount of trials of respectively kind to acquire acceptable signal-tonoise improvement finished signals be around. The number of trials required will differ dependent on the density of the incentives and is usually additional for a field situation.

14. Usage suitable scientific and numerical performance to analyses the facts. Do not organize the reactions conferring to biased decisions. Use numerical actions correctly and practically.

15. Use a scientific organization algorithm, such as bootstrapping on associations, that separates the adaptable severe by categorizing the reactions to the investigation incentives as presence moreover more comparable to the physical reactions or the unrelated responses.

16. Use an accurate data investigation algorithm that precedes into interpretation the unpredictability through particular trials, such as bootstrapping.

17. Usual an exact, sensible numerical measure for facts existing determination and a distinct, precise, sensible numerical measure for facts inattentive determination. Categorize results that do not meet also principle as unclassified. Identify that unspecified consequence is not a fault, neither a false negative nor a false positive.

18. Control technical assumptions to a purpose as to whether or not a focus has the precise situation pertinent knowledge in-person investigations stored in his mind. Distinguish that mind fingerprinting notices only occurrence or nonexistence of facts that not responsibility, morality, any non-action or lying.

19. Ground fact is whether the detailed facts tested are in detail, stowed in the focus's mind. Usual active ground truths with declaration finished post-test discussions in research laboratory investigates and in-ground tests wherein questions are supportive. Create ground truth in so far as conceivable done unimportant means in real existence criminal requests with unhelpful questions. Differentiate that ground truth is the correct formal of what the focus in detail recognizes, not whether the topic is deceptive or guilty.

20. Variety technical resolves grounded on mind reactions. Do not challenge to kind technical resolves based on apparent actions that can be operated, such as response time. 


\section{Analysis}

\subsection{Enlightening evidence detection}

The recognition of mask evidence stored in the minds of defendants, eyewitnesses, intellect sources, and so on, is a concern to individual points of law administration, government and private surveys, and intellect actions. This system identifies info straight, on the source of the electrophysiological appearances of info handling mind action measured non-invasively from the scalp. Since it is contingent individual on mind info handling, it doesn't depend on the responsive of the issues.

\subsection{Mind mermer}

It develops complicated MERA to notice info stored in the human mind. A MERMER is elicited after a specific identifies and processes an arriving incentive that is important. When an unrelated incentive is realized, and the MERMER reaction is absent. The MERMER happens inside around a subsequent stimulus presentation and can be noticed.

\subsection{Scientific technique}

An arrangement of words or pictures is obtainable on audio-visual display under computer controller. Each incitement acts for a part of a second. There are 3 types of incentives, and they are: "irrelevant," "targets," and "probes". The goals are finished applicable and notable to all subjects. The subject has specified a list of the goal incentives and initiated to press a specific key in reaction to goals.

\subsection{Computer controlled}

The Mind Fingerprinting is under computer mechanism together with planning of the incentives and recording of electrical mind actions as well as a mathematical information investigation.
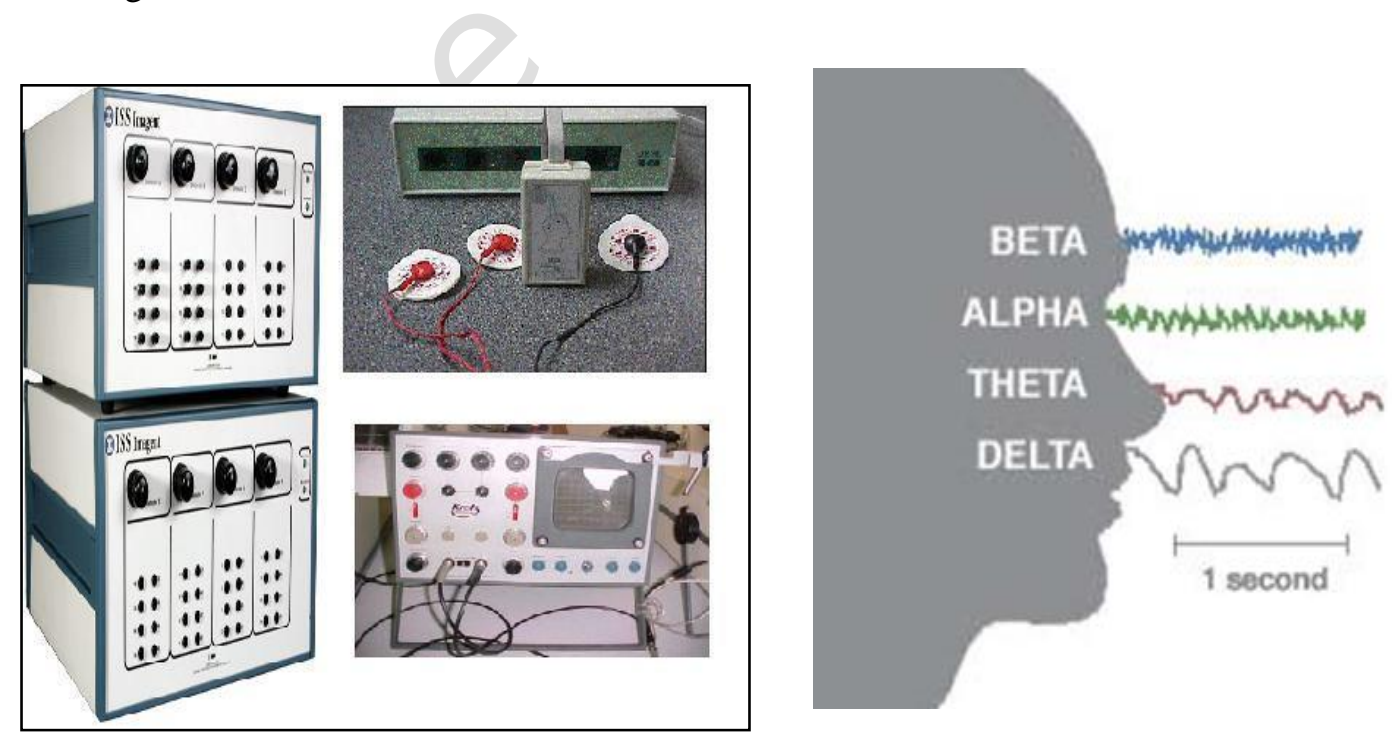

Figure 4. Mind Fingerprinting 


\subsection{Working procedure}

The individual to be tested wears distinctive head bandwidth electronic sensors that measure the electroencephalography from various locations on the scalp, as shown in below Figure 3. Mind fingerprinting uses reasoning mind responses and do not rest on the reactions of the subject, nor it precious by emotive responses. Mind fingerprinting is diverse from the lie-detector, which trials reaction-based physical signals.

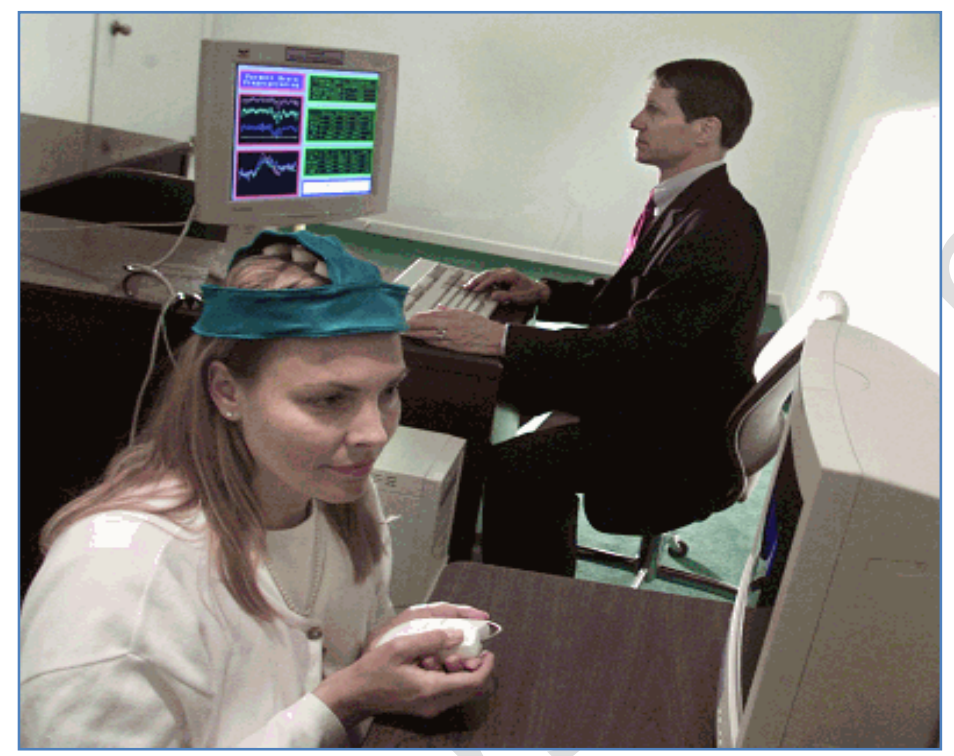

Figure 5. A person being tested irksome a particular headband with electrical devices

A Suspicious is verified by three categories of info represented by using diverse coloured lines:

1) Red: Facts the suspicious is predictable to recognize.

2) Green: Facts not recognized to suspicious.

3) Blue: Facts of the criminality that individual criminal would recognize.

\section{Conclusion}

Mind Fingerprinting is an innovative, fresh systematic technology knowledge for resolving corruptions, crimes, recognizing criminals, and absolving acquitted suspects by a best-ever of hundred percentage accurateness in investigation with United States management and government organizations, authentic illicit and criminal cases, and further claims. This skill achieves a crucial requirement for law prosecution organizations, supervisions, detectives, corporations, falsely suspects, acquitted accused and crime victims. Result of associated mind potentials for fault rate /accurateness and statistical assurance in four studies. 76 tests discovered existence or nonappearance of info concerning (1) real-life proceedings as well as offence crime; (2) actual crime with significant penalty (3) facts distinctive to Federal Bureau of Investigation (FBI) agents and (4) facts inimitable to explosive Bomb disposal experts. Among together P300-MERMER and P300, the faulty rate was zero percentage: calculations were a hundred per cent perfect, no false positive or false negative; and no in calculations. Counter procedures have no outcome. Average statistical assurance for calculations was $99.6 \%$ among P300 and $99.9 \%$ among P300-MERMER. Mind fingerprinting method and technical 
values for research laboratory and turf, field applications were discussed. Critical differences in methods that generate different results are recognized. Noticeably diverse methods in further studies have formed over 10 time's higher faulty rates and noticeably inferior statistical confidence. Facts maintain the assumptions to facilitate accurateness, consistency, and legality on subsequent the mind fingerprinting technical values outline in this.

\section{References}

[1] Jackson Olive Aluri, "Brain fingerprinting," International Journal of Advanced Research in Computer Science and Software Engineering, vol.5, no.1, (2015)

[2] BR Nivedhini, A Periyanayaki, "Brain fingerprinting technology," Psychology Imperial journal of interdisciplinary research, (2017)

[3] MJ Blitz, "Lie detection, mind reading, and brain reading," Searching Minds by Scanning Brains, pp.45-58, (2017)

[4] P McGorrery, "A further critique of brain fingerprinting: The possibility of propranolol usage by offenders," Alternative Law Journal, vol.42, no.3, pp.216-220, (2017) DOI: 10.1177/1037969X17730204

[5] HM Mallikarjun, "Machine learning-based classifier for falsehood detection," IOP Conference Series: Materials Science and Engineering, (2017)

[6] Kai Zhang, N.Thirupathi Rao, Debnath Bhattacharyya, "Matrix Depiction Based Cyst Detection in Pediatric Aged MRI/ ULTRASONIC and Ultrasonic Images," International Journal of Smart Home, vol.12, no.4, pp.27-38, (2018) DOI:10.21742/IJSH.2018.12.4.04

[7] Basar-Eroglu C, Basar E, and Demiralp T, "Schumann M P300-response: possible psychophysiological correlates in delta and theta frequency channels. A review," IJP, vol.13, no.2, pp.161-79, (1992)

[8] Harrington v. State, Case No.PCCV 073247, Iowa District Court for Pottawattamie County, March 5, (2001)

[9] Dongyue Wang, Taegkeun Whangbo, "3D Point Cloud Reconstruction Using Structure from Motion with Sequence Images in Hadoop," International Journal of Image and Signal Systems Engineering, vol.3, no.1, pp.1-6, (2019) DOI:10.21742/IJISSE.2019.3.1.01

[10] William f. leo, "Fingerprint identification: objective science or subjective opinion?" California, (2001) 